\title{
Légitimités et circulations des discours entre espace médiatique et espace politique : le cas d'une coopération salariale
}

The Circulation of Discourses Between the Media and the Political Sphere: The

Case of Wage Cooperation

\section{Christelle Chauzal-Larguier et Sébastien Rouquette}

\section{(2) OpenEdition}

Journals

Édition électronique

URL : http://journals.openedition.org/edc/6638

DOI : $10.4000 /$ edc. 6638

ISSN : 2101-0366

Éditeur

Université Lille-3

\section{Édition imprimée}

Date de publication : 1 décembre 2016

Pagination : 71-90

ISBN : 978-2-917562-16-1

ISSN : $1270-6841$

\section{Référence électronique}

Christelle Chauzal-Larguier et Sébastien Rouquette, « Légitimités et circulations des discours entre espace médiatique et espace politique : le cas d'une coopération salariale », Études de communication [En ligne], 47 | 2016, mis en ligne le 01 décembre 2018, consulté le 19 avril 2019. URL : http:// journals.openedition.org/edc/6638; DOI : 10.4000/edc.6638 


\section{Légitimités et circulations}

des discours entre espace médiatique et espace politique :

le cas d'une coopération salariale

The Circulation of Discourses Between the Media and the Political Sphere: The Case of Wage Cooperation

Christelle Chauzal-Larguier

Université Clermont-Ferrand 2, EA 4647 Laboratoire Communication et solidarité christelle.larguier@univ-bpclermont.fr

Sébastien Rouquette

Université Clermont-Ferrand 2, EA 4647 Laboratoire Communication et solidarité sebastien.rouquette@univ-bpclermont.fr 
L'objectif de l'article est d'étudier la circulation de discours entre l'espace public médiatique et l'espace public politique à partir d'un terrain précis, celui de la coopération salariale, à travers le don de RTT (Réduction de temps de travail). Au terme de ce travail, il apparaît que le cadrage médiatique centré sur l'impact humain ne donne pas aux différentes parties prenantes une visibilité à défendre leur point de vue à la hauteur de leurs investissements et de leurs rôles. Alors que l'agenda politique se révèle prépondérant dans l'importance médiatique accordée à ces situations, les responsables politiques sont bien moins audibles que les salariés, ce qui favorise une imperceptible mais forte réduction des questions débattues.

Mots-clés : légitimité, circulation des discours, cadrage médiatique, coopération salariale.
The objective of this article is to study the circulation of discourses between the public media sphere and the political sphere. Our particular focus is on the issue of wage cooperation and the donation of paid leave time. Our analysis shows that the media's framing of this issue emphasizes the human impact of wage cooperation in a way that is not reflective of the role or level of engagement of the various stakeholders. While media highlight the political agenda, politicians are given much less coverage than employees. Such media bias tends to create an imperceptible but significant reduction of debate.

Keywords: legitimacy, discourse circulation, media framing, wage cooperation. 
Galinsky, Bond et Friedman (1993) ont mis en évidence que $40 \%$ des salariés qui assument des responsabilités envers des membres de leurs familles éprouvent des difficultés à concilier vie privée et vie professionnelle. Les solutions qui leur sont apportées se limitent encore couramment à des arrangements au sein de l'entreprise (Chasserio et Legault, 2005), plus avantageux financièrement que les congés prévus par l'État. Le Loarne-Lemaire, Sanseau et Smith $(2014,41)$ proposent de remplacer cette approche individuelle des problèmes familiaux par une approche relationnelle, au sens large, impliquant tous les acteurs ayant des liens variés avec le salarié concerné (employeur, proches, famille, amis...). La recherche de solutions impliquerait la mobilisation de différentes parties prenantes ${ }^{1}$. Le don de RTT de salariés au bénéfice d'un collègue touché par un problème de maladie grave dans sa famille est devenu un fait de société qui illustre cette approche relationnelle. C'est ensuite devenu un sujet médiatique quand ces dons ont commencé à faire l'objet d'articles à partir de 2009. C'est enfin une question politique quand ces dons de jours de congés - au départ spontanés et non encadrés par la loi - ont abouti à un encadrement législatif. Cet encadrement a donné lieu à une proposition de loi votée en première lecture le 25 janvier 2012, une loi votée par les deux chambres en $2014^{2}$ et un décret d'application au secteur public en $2015^{3}$.

Dès lors, comment les discours portés sur les actions entreprises par ces salariés et sur les notions de solidarité sous-jacentes circulent-ils entre l'espace médiatique et l'espace politique?

Prenant exemple sur un don de RTT, largement médiatisé, survenu en 2009 dans sa circonscription (le cas Badoit), un député de la majorité UMP de l'époque, Paul Salen, dépose une proposition de loi sur une mesure non coûteuse financièrement pour l'État et potentiellement avantageuse politiquement, la solidarité étant relativement consensuelle. Selon lui, " c'est un sujet où il n'y a plus ni droite ni gauche » (Europe 1, 18 août 2011). La loi ne sera votée que trois ans plus tard et les débats politiques qui ont lieu au parlement à cette occasion en 2014, entre majorité favorable à la loi et opposition socialiste et communiste, seront nourris de problèmes de fond (Faut-il légiférer sur un tel sujet ? Cela ne couvre-t-il pas un désengagement de l'État ?...) et de forme (Le don de RTT est-il une mesure égalitaire ? Est-ce aux salariés d'assumer le don ?...).

S'il est vrai que les problèmes publics sont toujours le résultat de conflits de significations entre groupes sociaux, idéologies, représentations différentes (Hall, 2007), l'intérêt de cet article est de voir par quels processus un sujet

1 Ce concept est entendu ici dans l'acceptation générale proposée par Freeman (1984) et concernant des acteurs détenant le pouvoir et/ou la légitimité et/ou intervenant en situation d'urgence (Mitchell, Agle et Wood, 1997).

2 Loi votée le 09 mai 2014 « visant à permettre aux salariés de faire don d'heures de réduction de temps de travail ou de récupération à un parent d'un enfant gravement malade » (article L.1225-65-1 du code du travail).

3 Décret d'application publié le 29 mai 2015 ( $\left.n^{\circ} 2015-580\right)$. 
conflictuel dans l'espace politique ne l'est plus dans l'espace médiatique. En étudiant la circulation des discours portés sur la coopération salariale entre ces deux espaces, l'objectif est de comprendre comment et pourquoi les opinions critiques développées au parlement ont été minorées dans la presse.

Pour cela, ce travail combine trois méthodes. II s'intéresse, en premier lieu, à la médiatisation des dons de RTT. Le corpus est constitué de 18 cas parmi les plus médiatisés ${ }^{4}$ entre 2009 et 2015 , et ce afin d'étudier les conflits sousjacents de cadrage et d'agenda dans leur durée. Il ne suffit pas de constater qu'un thème accède à la presse pour mesurer l'importance qu'on lui accorde. Il faut aussi s'intéresser à la manière dont la presse en parle. Car les effets de cadrage pèsent sur la manière dont les lecteurs apprécient le sujet (Mercier, 2012). 136 articles écrits ou reportages radiotélévisés ont traité de ces 18 dons. La liste de titres traitant de ces sujets est diverse ${ }^{5}$. Le corpus se compose de 12 articles de presse spécialisée (presse magazine), 45 synthèses ou reportages de presse quotidienne nationale, 44 de presse quotidienne régionale et enfin de 35 articles de blogs ou de sites internet (juridiques, de santé, de société, institutionnels, consacrés aux familles ou encore au management). La diversité des radios, télévisions, journaux, magazines, sites internet d'actualité et blogs, qui ont porté à la connaissance de tous les faits et les événements discutés collectivement, reflète la fragmentation ${ }^{6}$ de l'espace médiatique. Intégrer des reportages radios, télés et des articles de presse permet de tenir compte de la diversité des médias et des publics auxquels ils s'adressent (Devillard et Marchetti, 2008, 150-151). Le faire avec les sites et les blogs permet de prendre

4 Pour faciliter la compréhension, les 18 cas sont classés par ordre chronologique au regard de la date du vote de la loi sur le don de RTT et de son cadre législatif. Ainsi, 5 cas ont été médiatisés avant le vote de la proposition de loi de 2012 : Badoit (2009), TC2 (2009), Fuji-Autotech (2011), Groupe Pierre Guérin (2012), Conseil Général des Ardennes (2012). Cas du secteur public médiatisés avant la publication du décret : Policier de Nancy (2014), L’hôpital de Montaigu (2014), Agent SNCF d'Ille et Vilaine (2014), SNCF Auvergne (2013), Policiers de Rive-de-Gier (2015). Cas du secteur privé : Maison de retraite de Réalmont (2015), Omnistrans (2015), Verrerie Pochet du Courval (2015), LaM de Villeneuve-d'Ascq (2015), Leader Price (2015), DPS\&CO (2015). 2 cas postérieurs à la loi ne respectant pas le lien de descendance identifié par la loi : Traminots de Nice (2014), Gibaud (2015).

5 Dont : L'Est Républicain, Le Parisien, La Dépêche du Midi, Terra Fémina, Le Télégramme, Le Figaro, France Bleu, France 3 ; RFM, Le Post, France Soir, L'Obs, La Nouvelle République, L'Union, La Montagne, Ouest France, Complément-air, Nice Matin, 20 minutes, Le Point, Francesoir.fr, La Voix du Nord, Europe 1, Le Dauphiné Libéré, pourquoidocteur.fr, Métronews, Midi Libre, Managerattitude. fr, Pageactu.orange, Famili.fr, Pop-corn.news, Le Journal d'Ici, L'Express, M6, Métronews, Parents.fr, Radioscoop, Positiv.fr, Sud-ouest, Auféminin.com, Le Tribunal du net, l'info.re, Blog.elle.fr, Abc-bonheur, Provocateurdesourires. com, pausecafé.fr, Le Progrès, niooz.fr, T7, Normandie actu, L'Informateur, L'Éclaireur, Le Réveil de Neufchâtel, Oh my mag, Démotiveur.fr, Diav-tv.info, Magicmaman.fr, Sudinfo.be, Nord Éclair, L'Essor de la Loire, Le Pays roannais, Zoomd'ici.fr, Yahoo Paris Normandie, France Info.

6 L'espace public se caractérise par sa fragmentation croissante (Neveu, 1999), y compris sa partie médiatique en raison de la multiplication des sites d'information et des blogs (Rouquette, 2010). 
aussi en compte ces parties nouvelles de l'espace médiatique (Rouquette, op. cit., 2010, 16).

Ce cadrage médiatique est, en deuxième lieu, mis en perspective en étudiant le point de vue de parties prenantes à partir de l'interview d'un responsable politique ${ }^{7}$ (la sénatrice Catherine Deroche, rapporteure de la loi au Sénat) et d'une journaliste du quotidien régional La Montagne ${ }^{8}$ (ayant publié le 29 avril 2014 un article sur le don de RTT intervenu à la SNCF Auvergne). Ces entretiens ont été menés avec un guide organisé autour de la médiatisation du don de RTT (conditions, raisons, contenu, perceptions). Étudier le point de vue, le ressenti et les arguments des acteurs voulant assurer la promotion de ces faits (Cobb et Ross, 1997) apporte un éclairage sur la perception que ceux-ci ont de leurs actions. Cela permet aussi d'analyser simultanément la phase de construction de la conciliation travail/famille comme problème public et sa phase de circulation médiatique " afin de prendre en compte l'indissociabilité du processus de communication » (Comby et Grossetête, 2007, 1).

Ce cadrage médiatique est, en troisième lieu, comparé au traitement politique de cette question. Nous avons utilisé pour cette recherche les comptes rendus des débats de la commission des affaires sociales de l'Assemblée nationale $^{9}$ (20 pages) et du Sénat ${ }^{10}$ (26 pages). Il faut pour cela préciser les différences existant entre l'espace politique et l'espace public médiatique. Ce dernier est un espace de discussion dans lequel se confrontent les discours tenus par différents acteurs d'une société. Il fait partie des instances qui, à la fois, portent à la connaissance de tous les problèmes traités publiquement, gèrent et orientent cette attention en sélectionnant les sujets rendus publics et participent à l'organisation des débats entre citoyens sur ces sujets (Quéré, 1995, 12). Il est ici constitué d'articles de presse, de sujets télés et radios, de billets publiés sur les blogs ${ }^{11}$ traitant de dons de RTT. L'espace public médiatique se distingue de l'espace politique dans la mesure où, dans ce dernier, il ne s'agit plus seulement de délibérer et de discuter mais de décider des

7 Entretien réalisé le 08 février 2016.

8 Entretien réalisé le 04 mars 2016.

9 «Rapport fait au nom de la commission des Affaires sociales sur la proposition de loi visant à permettre aux salariés de faire don d'heures de réduction de temps de travail ou de récupération à un parent d'un enfant gravement malade ». Assemblée nationale, mis en ligne le 18 janvier 2012, consulté le 19 février 2016. URL : http://www.assemblee-nationale.fr/13/rapports/r4179.asp.

10 « Rapport fait au nom de la commission des Affaires sociales sur la proposition de loi, adoptée par l'Assemblée nationale, visant à permettre le don de jours de repos à un parent d'un enfant gravement malade ». Sénat, mis en ligne le 16 avril 2014, consulté le 23 avril 2016. URL : https://www.senat.fr/rap/ 113-456/113-456.

11 Seule une partie d'internet prend part à l'espace médiatique, c'est le cas des sites internet, blogs, pages en ligne sur lesquels les internautes se connectent pour suivre ou comprendre l'actualité et qui leur donnent accès à l'information et aux débats d'actualité (Rouquette, op. cit., 2010). 
affaires de la cité et d'agir. L'espace politique est lié à la notion de pouvoir. II est constitué ici des débats politiques qui se sont tenus au parlement et des décisions législatives sur le don de RTT qui en découlent, décisions - c'est l'un des enjeux supplémentaires de l'espace public politique par rapport à l'espace public médiatique (Wolton, 1997) - applicables sur tout le territoire national.

\section{1.}

\section{L'agenda politique prépondérant pour la promotion des discours de coopération salariale}

La circulation des discours entre l'espace public médiatique et l'espace politique va d'abord être étudiée sous l'angle de la promotion de ces cas de coopération salariale : quels acteurs, quelles parties prenantes, se révèlent centrales dans la constitution de ces faits de société en problèmes discutés publiquement?

\subsection{Le rôle médiatique des salariés en apparence central}

Le père de Mathys, ayant bénéficié d'un don de 170 jours de RTT en 2009 de la part de ses collègues de la société Badoit, est un précurseur dans la publicisation d'un problème d'abord privé. Entre 2011 et 2014, 24 sujets, exclusivement consacrés au cas, sont publiés mobilisant différents supports médiatiques (presse quotidienne locale et nationale, revues généralistes et spécialisées, radio, télévision, journaux en ligne).

L'action des salariés du cas Badoit suffit-elle à expliquer l'importance médiatique accordée à ces questions ? En effet, la médiatisation ne va pas de soi, ne serait-ce que parce que les bénéficiaires ne souhaitent pas forcément que leur histoire soit médiatisée dans un contexte familial complexe. Si la mobilisation des collègues donateurs est indispensable, elle reste largement dans l'ombre, souvent pour respecter leur volonté, comme l'avoue la journaliste interrogée pour cette recherche : "Le contact est rare avec les collègues. Ces derniers préfèrent ne pas communiquer ».

En outre, cette médiatisation dépend notamment de facteurs extérieurs, antérieurs ou postérieurs aux actions médiatisées (Neveu, 1999). Ainsi, les parents de Mathys ont la volonté d'entretenir l'intérêt pour que le processus de légalisation des dons aille à son terme. Ils sollicitent le député Paul Salen pour que soit entamé un travail législatif. La même démarche est ensuite entreprise par les parents d'Éva, huit ans (cas du policier de Nancy, 2014) ou la maman de Nicolas, dix-sept ans et atteint d'un cancer (cas de l'aide-soignante de l'hôpital de Montaigu, 2014). Agents du secteur public, ils médiatiseront leur histoire (presse, radio et réseaux sociaux dans le premier cas, presse dans le second), mettant en évidence qu'ils ont obtenu des dons de jours de RTT de leurs collè- 
gues sans pouvoir en bénéficier, en l'absence de vote du décret d'application. En effet, dans les deux cas, n'ayant pas de texte de loi spécifique au secteur public sur lequel s'appuyer, les supérieurs hiérarchiques de ces parents ont refusé la mise en place du don. "Comme il n'existe toujours pas [de décret], on ne sait pas davantage aujourd'hui [décembre 2014] comment cela doit s'appliquer à nos salariés, qui y ont pourtant droit puisque c'est la loi » admettait, à l'époque, Catherine Daries, directrice d'hôpital dans les Hautes-Pyrénées (Aujourd'hui en France, 9 décembre 2014). Pour accroître l'attention portée à ces discours tenus par des anonymes, défenseurs d'un geste citoyen, un relais médiatique exercé par des responsables politiques de terrain est décisif. Ainsi, le député Paul Salen (187 citations dans la presse générale et spécialisée écrite, télévisuelle, radio, blogs et sites internet), porteur de la proposition de loi, la sénatrice Catherine Deroche (67 citations), rapporteure du texte de loi sur le don de RTT, et Marylise Lebranchu (82 citations), ministre de la Fonction publique ayant soutenu le vote du décret d'application aux corps de l'État, ont défendu la pertinence des actions entreprises sur le terrain en y faisant tous référence à deux reprises sur leurs blogs.

\subsection{La primauté de l'agenda politique}

Plusieurs articles de presse, reportages audiovisuels et billets en ligne consacrent notamment une attention à ces coopérations salariales au moment des étapes clés du processus législatif : le dépôt de la proposition de loi (2011), le vote de la loi à l'Assemblée nationale (2012) puis au Sénat (2014) et la publication officielle du décret d'application à la fonction publique (2015). Ainsi, entre 2012 et 2014, cela correspond à 68 articles, reportages et billets, sur les 136 de l'ensemble du corpus. À l'inverse, cette médiatisation est faible avant les débats législatifs. Entre 2009 et 2011, 20 articles, reportages et billets en ligne, sont consacrés à ces questions. Cette médiatisation a ensuite décru rapidement avec 48 articles publiés entre fin 2014 et fin 2015.

Avec ce nombre important de productions médiatiques, principalement entre 2012 et 2014, la presse cherche à illustrer, voire à faire avancer, les débats parlementaires : "C'est notre modeste contribution pour faire avancer les choses " avouera la journaliste interviewée. Autrement dit, c'est l'agenda médiatique qui s'adapte à l'agenda politique, non l'inverse. Il faut alors s'intéresser aux motivations des principales parties prenantes responsables du traitement législatif de ces questions. La sénatrice Catherine Deroche explique qu'elle-même a été sollicitée par son collègue député : "Paul Salen avait déposé sa proposition de loi. Pour être effectif, il faut que cela soit vu par le Sénat. II m'a sollicitée pour que le projet avance et soit étendu notamment à la fonction publique ". Ses motivations relèvent donc avant tout de la gestion de la vie de la cité : «Le don de RTT permet au bénéficiaire de profiter de jours mieux rémunérés car la maladie occasionne en plus des charges financières supplémentaires " et correspond à un réel besoin (1 500 familles seraient potentiellement concernées) selon le député (Le Monde, 19 décembre 2014). 
L'importance de contrôler de telles démarches solidaires permet de justifier le rôle des responsables politiques comme nous l'affirme Catherine Deroche : «La loi était utile pour mettre des garde-fous. L'objet du don notamment. Il fallait préciser. On ne voulait pas que des gens donnent tous leurs jours de congés ».

L'initiative des médias dans l'agenda collectif est par conséquent limitée. Certes, la mobilisation des salariés et des parents est incontournable. Pourtant, la médiation d'un député qui juge politiquement opportun de se saisir de cette question se révèle centrale. L'intervention de responsables politiques se produira deux nouvelles fois (avec la sénatrice et la ministre de la Fonction publique) pour faire avancer le processus législatif. Ils n'hésiteront d'ailleurs pas à intervenir dans les médias. Il faut alors certes considérer les médias comme l'un des participants du processus de mise à l'agenda, mais ce calendrier montre, qu'en ce domaine, les médias jouent " plus souvent un rôle de relais par rapport à des problèmes portés par d'autres acteurs qu'un rôle d'initiateur » (Hassenteufel, 2010, 52), relativisant - ici comme ailleurs - la thèse de la primauté des médias dans la hiérarchisation des problèmes considérés comme importants par la population.

Pour les médias, l'intérêt à promouvoir ces coopérations salariales comme problèmes publics est élevé tant qu'il s'agit de situations hors la loi. Cet intérêt se réduit dès que le texte de loi et le décret entrent en vigueur. On voit bien l'importance du traitement juridique des dons, et donc de l'agenda politique dans la médiatisation de cette cause, à cette remarque de la journaliste interrogée : "Maintenant [2016], le rôle du journaliste est moins pertinent voire même discutable. Le journaliste n'a plus rien à apporter. II ne surprend plus son lectorat ".

Au final, l'intervention des responsables politiques pour ériger ces faits de société en questions publiques se révèle prépondérante. Les médias ontils pour autant traité de ces faits comme d'une question politique ? La place accordée par les médias au cadrage politique est-elle à la hauteur du rôle joué par les élus dans la promotion de ces actions ? Cela implique de donner une importance significative aux dimensions idéologiques et morales de ces questions et une visibilité aux commentaires des responsables politiques. Sur le terrain, un tel état de fait n'est pas, comme nous allons le voir, respecté. 
2.

\section{Le cadrage médiatique de la coopération salariale : la minoration de la dimension politique}

\subsection{Du commentaire législatif au traitement social}

L'étude du cadrage médiatique est une démarche balisée dans le champ de l'analyse des médias. Elle vise au fond à répondre aux questions suivantes : sous quel angle ce sujet est-il traité, dans quel régime de problèmes est-il inscrit (Mainil, 2015, 10) ?

Pour y répondre, il faut établir une typologie des cadres médiatiques utilisés ici. La typologie de Neuman, Just et Crigler a servi de première référence. Leur recherche distingue quatre cadres : le cadre de conflit, les conséquences économiques, l'impact humain et le cadre de moralité (Neuman, Just et Criglier, 1992). Une approche exploratoire a permis de vérifier l'utilité de cette typologie à la condition suivante : adapter ces cadrages aux sujets abordés dans nos articles et faire évoluer en conséquence les critères adoptés (tableau 1).

\begin{tabular}{|c|c|}
\hline Cadrage privilégié & \multicolumn{1}{c|}{ Critères } \\
\hline $\begin{array}{c}\text { Responsabilité/ } \\
\text { solution }\end{array}$ & $\begin{array}{l}\text { Le récit médiatique rapportant ces coopérations salariales } \\
\text { suggère-t-il un responsable de la situation (par exemple } \\
\text { ici : suggère-t-il que le gouvernement, les entreprises sont } \\
\text { responsables de ces situations empêchant les parents } \\
\text { d'enfants malades de rester à leurs chevets)? } \\
\text { Suggère-t-il de légaliser des pratiques de solidarité } \\
\text { existantes? }\end{array}$ \\
\hline $\begin{array}{c}\text { Impact humain } \\
\text { (Dimension humaine) }\end{array}$ & $\begin{array}{l}\text { Les articles et reportages publient-ils des témoignages de } \\
\text { soutien, d'aide, de détresse personnelle (témoignages des } \\
\text { maladies, fatigue, courage de l'enfant) de sorte à générer des } \\
\text { sentiments d'empathie, de sympathie ou de compassion? }\end{array}$ \\
\hline $\begin{array}{c}\text { Jugement } \\
\text { (moral et/ou politique) }\end{array}$ & $\begin{array}{l}\text { Les sujets font-ils référence à une certaine moralité ou à des } \\
\text { jugements de valeur, discutent-ils de la justesse de la loi et } \\
\text { jugent-ils de la valeur des comportements commentés? }\end{array}$ \\
\hline $\begin{array}{c}\text { Conséquences } \\
\text { économiques }\end{array}$ & $\begin{array}{l}\text { Est-il fait mention de pertes financières des familles } \\
\text { concernées ou des dépenses générées par ces situations? } \\
\text { Est-il fait mention des aspects économiques de la loi ? }\end{array}$ \\
\hline
\end{tabular}


L'application de ces critères aux 136 articles écrits ou reportages radiotélévisés étudiés donne les résultats suivants :

\begin{tabular}{|c|c|c|c|c|c|}
\hline & \multicolumn{4}{|c|}{$\begin{array}{l}\text { Cadrage médiatique de l'article ou du reportage } \\
\text { (en \%) }\end{array}$} & \\
\hline & $\begin{array}{l}\text { Responsabilité/ } \\
\text { solution }\end{array}$ & $\begin{array}{l}\text { Impact } \\
\text { humain }\end{array}$ & Jugement & $\begin{array}{l}\text { Conséquences } \\
\text { économiques }\end{array}$ & \\
\hline Total & $64 \%^{12}$ & $63 \%$ & $27 \%$ & $10 \%$ & $\begin{array}{l}\text { Corpus } \\
\text { de } 136 \\
\text { articles }\end{array}$ \\
\hline $\begin{array}{l}\text { Badoit (commenté en } \\
2009 \text {, mais surtout } \\
2012 \text { et 2014) }\end{array}$ & $100 \%$ & $41 \%$ & $44 \%$ & $15 \%$ & 39 art. \\
\hline Fuji-Autotech, 2011 & $50 \%$ & $71 \%$ & $21 \%$ & $7 \%$ & 14 art. \\
\hline $\begin{array}{l}\text { Groupe Pierre Guérin, } \\
2012\end{array}$ & & $100 \%$ & $33 \%$ & $33 \%$ & 3 art. \\
\hline Ardennes, 2012 & & $100 \%$ & & & 2 art. \\
\hline $\begin{array}{l}\text { SNCF Auvergne, } \\
\text { 2013-2014 }\end{array}$ & $33 \%$ & $66 \%$ & & & 3 art. \\
\hline $\begin{array}{l}\text { Policier de Nancy, } \\
2014\end{array}$ & $90 \%$ & $61 \%$ & $38 \%$ & $24 \%$ & 21 art. \\
\hline $\begin{array}{l}\text { SNCF d'Ille-et-Vilaine, } \\
2014\end{array}$ & $100 \%$ & $100 \%$ & $100 \%$ & $100 \%$ & 1 art. \\
\hline $\begin{array}{l}\text { Hôpital de Montaigu, } \\
\qquad 2014\end{array}$ & $100 \%$ & $100 \%$ & $20 \%$ & & 5 art. \\
\hline $\begin{array}{l}\text { Traminots de Nice, } \\
2014\end{array}$ & & $100 \%$ & & & 15 art. \\
\hline Réalmont, 2015 & & $100 \%$ & & & 1 art. \\
\hline Omnitrans, 2015 & $10 \%$ & $90 \%$ & & & 10 art. \\
\hline Rive-de-Gier, 2015 & & $100 \%$ & & & 3 art. \\
\hline
\end{tabular}

12 Un article peut développer des paragraphes relevant de cadres différents: plusieurs réponses sont donc possibles. 


\begin{tabular}{|c|c|c|c|c|c|}
\hline $\begin{array}{c}\text { Pochet du Courval, } \\
2015\end{array}$ & $66 \%$ & $100 \%$ & & & 3 art. \\
\hline $\begin{array}{c}\text { Villeneuve-d'Ascq, } \\
2015\end{array}$ & & $100 \%$ & & & 1 art. \\
\hline Leader Price, 2015 & & $100 \%$ & & & 1 art. \\
\hline DPS\&CO, 2015 & $89 \%$ & $89 \%$ & $66 \%$ & & 2 art. \\
\hline Gibaud, 2015 & & $100 \%$ & & & 9 art. \\
\hline Hôpital Isère, 2015 & & & & 3 art. \\
\hline
\end{tabular}

Tableau 2 : l'évolution du cadrage du don de RTT entre 2009 et 2015

La primauté de l'agenda politique devrait se traduire par une primauté accordée aux explications des tenants et aboutissants juridiques et moraux de la loi. II n'en est rien. Comme le montre le tableau 2, le traitement médiatique des dons de RTT favorise deux cadrages :

celui de la responsabilité (une loi inadaptée) et des solutions (légiférer) (64\% des 136 articles publiés développent ainsi le cadrage « responsabilité/solution » (soit 88 articles sur 136),

l'angle individuel (la détresse des parents, le courage des enfants : cet angle concerne $63 \%$ des sujets publiés).

À l'inverse, le cadrage politique (le jugement politique) et économique (les coûts et bénéfices économiques de la loi) est minoré. Plusieurs explications peuvent être avancées pour expliquer ces choix médiatiques implicites.

La première est de raisonner ainsi : à quelle période le cadrage politique est-il prépondérant ? Principalement quand le caractère illégal des actions de solidarité en fait un " bon objet ». Le caractère surprenant de l'information (la solidarité illégale) la rend intéressante. Les journalistes peuvent en effet supposer que beaucoup de lecteurs/salariés constatent une absence de solidarité dans leur quotidien et qu'une telle solidarité dissone avec l'affaiblissement des collectifs de travail qui caractérise l'évolution des relations professionnelles quotidiennes.

Ces arguments critiques ne sont, en deuxième lieu, repris dans la presse qu'au moment des discussions autour de la loi, autrement dit dans les périodes où l'agenda politique justifie - du point de vue des médias - un traitement politique de cette actualité. Il serait possible de n'y voir qu'un excès de prudence, cette option caractérisant particulièrement les titres régionaux. Des titres qui se signalent par une volonté de toucher la population d'une région, tous bords politiques confondus, quitte à privilégier l'information positive de proximité. 
De ce point de vue, les manifestations organisées autour des dons de RTT (marches, repas, etc.) ont le double avantage de valoriser l'action locale sans " crainte de prendre parti entre des composantes du terroir ou de la cité qui peuvent aussi être des lectorats » (Neveu, 2015, 173).

Mais il faut aussi, en troisième lieu, considérer que le traitement dépolitisé de sujets de société est la conséquence d'un positionnement de plus en plus dépolitisé des journaux eux-mêmes. Comme le constate Juhem $(2001,113)$, la revendication de neutralité des journaux implique qu'ils ne peuvent plus engager en leur nom une « proposition politique sous peine de voir leur rédaction mise en cause comme 'engagée' et 'politisée' ». Les journalistes sont amenés, par la logique de leur position professionnelle, à circonscrire leur activité à un commentaire des offres politiques existantes » (Juhem, 2001). Le cadrage politique des coopérations salariales aurait été facilité si les partis de l'opposition avaient fait de cette question un enjeu politique majeur, multipliant les critiques et les prises de position politiques. Ce n'est pas le cas, la priorité de l'opposition dans cette période de campagne présidentielle (1er semestre 2012) étant de dénoncer les politiques économiques du gouvernement. C'est pourquoi - comme le montre le tableau 2 - les journaux privilégient un traitement « factuel » plutôt qu'éditorial de ce dossier. Même si ce traitement «social » de la coopération salariale ne s'abstrait pas de jugements implicites (valorisation des solutions initiées par des acteurs privés [collègues, amis] gérées directement au sein des entreprises).

\subsection{Vers l'amoindrissement de la dimension politique des débats}

Or, cette évolution du cadrage médiatique se traduit par une réduction des termes du débat. Il suffit de s'intéresser aux débats législatifs pour s'apercevoir que les dons de RTT ont pourtant soulevé des interrogations dans l'espace politique. Pour mesurer l'importance de ce réductionnisme discursif et argumentatif, deux documents peuvent servir de référence à cette comparaison : les extraits du débat de la commission des affaires sociales de l'Assemblée nationale et du Sénat. Ces comptes rendus permettent de saisir les réserves politiques, économiques ou morales émises lors de l'examen de la proposition de loi et reprises lors de l'examen du texte de loi. Par exemple, les contreparties de la dimension charitable de la loi sont questionnées par plusieurs parlementaires: le député PS Michel Issindou se demande ainsi « pourquoi ne pas recourir au droit du travail, plutôt qu'à la bonne volonté collective [...] même si leur anonymat sera préservé, les collègues d'un salarié dont l'enfant est gravement malade [...] se sentiront plus ou moins tenus de faire un geste $»^{13}$. Le caractère inégalitaire de la situation des salariés est souligné par des élus de l'opposi-

13 Rapport de la commission des affaires sociales de l'Assemblé Nationale du 18 janvier 2012. 
tion : pour Jacqueline Fraysse, députée communiste ${ }^{14}$, "les salariés n'ont pas droit au même nombre de jours selon qu'ils travaillent dans une PME ou dans une grande entreprise, ce qui crée une inégalité ". Dans un autre registre, le rattachement du don de RTT à la solidarité est remis en cause par la sénatrice écologiste Aline Archimbaud ${ }^{15}$ : "Nous sommes dans le domaine de l'entraide, du care ; il s'agit de générosité, pas de solidarité ».

La pertinence de légiférer sur la solidarité divise tant à l'Assemblée nationale qu'au Sénat, y compris chez les députés approuvant la démarche collective des salariés. "Faut-il lui donner force de loi ? " s'interroge Jean-Patrick Gille, député PS, pour qui «transformer un geste de solidarité en disposition législative risque de lui faire perdre son aspect le plus positif, qui tient à son caractère spontané ${ }^{16}$. Car, « il est toutefois paradoxal de légiférer sur des comportements qui devraient être naturels " s'indigne Catherine Génisson, sénatrice $\mathrm{PS}^{17}$.

Au final, lors du vote de la proposition de loi, le texte est soutenu par les 136 députés de la droite et du centre, les socialistes se sont en effet abstenus et les communistes tout comme le Front de gauche s'y sont opposés. Les mêmes clivages politiques se retrouvent au Sénat, chaque parti politique campant sur ses positions.

Mais, de fait, le contenu de ces discussions politiques n'est pas systématiquement repris dans les articles publiés lors du vote de la loi (tableau 3). 


\begin{tabular}{|c|c|c|c|c|}
\hline & Titre de l'article & $\begin{array}{l}\text { L'article } \\
\text { mentionne-t- } \\
\text { il les résultats } \\
\text { du vote } \\
\text { au Sénat } \\
\text { (favorable, } \\
\text { défavorable, } \\
\text { abstention)? }\end{array}$ & $\begin{array}{l}\text { L'article } \\
\text { détaille- } \\
\text { t-il les } \\
\text { arguments } \\
\text { des } \\
\text { sénateurs } \\
\text { opposants } \\
\text { ou s'étant } \\
\text { abstenus? }\end{array}$ & $\begin{array}{l}\text { L'article } \\
\text { détaille- } \\
\text { t-il les } \\
\text { arguments } \\
\text { favorables } \\
\text { à la loi de } \\
\text { la sénatrice } \\
\text { rapporteure } \\
\text { du projet? }\end{array}$ \\
\hline L'Express & $\begin{array}{l}\text { Le don de congés, l'idée } \\
\text { " charitable » qui agace la } \\
\text { gauche. }\end{array}$ & $X$ & $X$ & $X$ \\
\hline Le Monde & $\begin{array}{l}\text { Le don de RTT à un } \\
\text { collègue dont l'enfant est } \\
\text { malade autorisé par le } \\
\text { Sénat. }\end{array}$ & $X$ & $x$ & $X$ \\
\hline $\begin{array}{l}\text { Le Nouvel } \\
\text { Obs }\end{array}$ & $\begin{array}{l}\text { Don de RTT : } 3 \text { questions } \\
\text { sur une avancée attendue. }\end{array}$ & $X$ & & $x$ \\
\hline Le Parisien & $\begin{array}{l}\text { Le don de RTT bientôt } \\
\text { légal. }\end{array}$ & $X$ & & $x$ \\
\hline La Croix & $\begin{array}{l}\text { Les salariés pourront } \\
\text { bientôt faire don de jours } \\
\text { de repos. }\end{array}$ & $X$ & & $X$ \\
\hline Direct Matin & $\begin{array}{l}\text { Le don de RTT : comment } \\
\text { cela va marcher? }\end{array}$ & $X$ & & \\
\hline 20 minutes & $\begin{array}{l}\text { Donner ses RTT à un } \\
\text { collègue dont l'enfant est } \\
\text { malade, c'est maintenant } \\
\text { possible. }\end{array}$ & $X$ & $\mathrm{X}$ & $x$ \\
\hline
\end{tabular}

Tableau 3 : comparatif des structures des articles de la presse nationale du 30 avril 2014 
Sur les sept quotidiens nationaux du corpus, si tous les journaux rappellent les résultats du vote et donnent - sauf Direct Matin - les arguments en faveur de la loi, seuls trois (L'Express, Le Monde et 20 minutes) citent les arguments des sénateurs opposants ou abstentionnistes.

Une place prépondérante est accordée par les médias aux trois acteurs politiques majeurs dans ce dossier (Paul Salen, Catherine Deroche et Marylise Lebranchu) confortés par d'autres acteurs de second plan ${ }^{18}$ ralliés à leur cause.

À l'inverse, des opposants avouent avoir eu des difficultés à se faire entendre : «On nous a accusés d'être des méchants, incapables d'humanité et de générosité. On a subi une pression folle » explique Dominique Watrin, sénateur communiste (L'Express Emploi, 30 avril 2014). Pourtant, des arguments sont défendus dans les grands médias. Comme le rapporte le Huffington Post (25 janvier 2012), Roland Muzeau, porte-parole des députés communistes, prévient par exemple que " le défaut du dispositif existant est qu'il ne complète pas suffisamment le salaire. II faut que les employeurs soient mis à contribution. II n'est pas juste de demander aux salariés de répartir les difficultés entre eux ».

Les rares critiques politiques reprises par les médias ne l'ont été que dans une période limitée - uniquement au moment des discussions à l'Assemblée et au Sénat - et sont souvent le fait de quotidiens d'opposition. Ce même sénateur communiste, Roland Muzeau, souligne les insuffisances du dispositif voté :

Aujourd'hui, I'Allocation journalière de présence parentale, prévue par le code de la sécurité sociale, implique une perte de salaire pour les parents contraints d'y recourir. C'est cette anomalie à laquelle il faut mettre un terme, en faisant obligation aux chefs d'entreprise d'assurer au salarié appelé à rester aux côtés de son enfant gravement malade, le maintien de ses revenus, et cela tout au long du traitement (L'Humanité, 22 janvier 2012).

Une fois le texte législatif acté, le traitement majoritairement accordé se restreint à la description des cas individuels. Cette tendance se traduit alors par une plus grande prise de distance avec les sources et les regards partisans sur des questions sociales : les parties prenantes politiques sont peu interviewées. Leur présence est conditionnée à un rôle effectif dans le processus législatif (le député Paul Salen, la sénatrice Catherine Deroche et le ministre de la Fonction publique, Marylise Lebranchu). Comme si les élus politiques étaient jugés, par les journalistes, peu légitimes pour porter un discours politique critique dans l'espace public médiatique sur ces questions.

18 Par exemple, le Huffington Post rapporte combien Marie-Anne Montchamp, secrétaire d'État aux Solidarités, se félicite du vote de la proposition de loi qui « valorise cet esprit de volontariat et de don sans lesquels nous ne pouvons espérer renforcer notre cohésion sociale " (Huffington Post, 25 janvier 2012). 
Or, sur un plan théorique, ce constat n'est pas sans intérêt. Cela signifie que les responsables politiques, malgré leurs connaissances des mécanismes de promotion médiatique de faits de société et leurs positions de législateurs, ne sont pas considérés comme les plus légitimes pour traiter des relations salariales privées et publiques. Et ce, malgré le principe selon lequel les « journalistes relaient plutôt les cadrages interprétatifs des promoteurs d'événements les plus légitimes, c'est-à-dire disposant de plus de pouvoir et de ressources symboliques » (Macé, 2005, 191). D'un côté, l'action législative des responsables politiques se révèle incontournable dans la promotion médiatique de ces coopérations, mais, d'un autre côté, leurs points de vue, explications et arguments à l'appui de ces actions législatives ne sont pas jugés incontournables dans les discussions relayées par la presse.

Or, cette minoration médiatique des points de vue politiques portés sur ces actions de coopération salariale n'a pas seulement pour effet d'écarter les acteurs politiques de ces débats. Elle a pour conséquence de laisser quasi uniquement la parole aux personnes favorables à ces textes de loi. Ces cadrages soulignent en effet l'importance grandissante accordée aux dimensions humaines et psychologiques de ces solidarités salariales.

Pourquoi - comme le montre le tableau 2 - les donateurs et les bénéficiaires des dons de RTT profitent-ils d'une grande exposition pour s'exprimer sur un tel sujet ? Pourquoi leur point de vue de parent d'enfant malade ou de collègue prime-t-il ? Simplement parce que ce sont les salariés qui sont précurseurs de cette démarche de coopération salariale d'un nouveau genre reposant sur une forte composante affective. La place accordée à leurs discours émane du fait qu'ils ont vécu le don de RTT de l'intérieur et qu'ils entretiennent une relation de proximité avec la personne, voire, le cas échéant, avec le problème rencontré par un collègue. Pour la journaliste interrogée, c'est aussi une obligation d'informer son lectorat sur une initiative alors méconnue ("Sinon les gens se demanderaient pourquoi ce n'est pas dans le journal, pourquoi nous ne sommes pas là »). Comptent désormais : les motivations des collègues, le ressenti des enfants et des parents bénéficiaires, la manière dont se sont enclenchées ces actions de solidarité (marches solidaires, dessins, etc.). De telle sorte que ces pratiques de solidarité disposent d'un très large écho dans la presse, quasiment sans discussion sur leurs prémisses idéologiques (solidarité entre collègues versus prestations sociales et familiales; charité individuelle versus solidarité collective). Ce constat en corrobore d'autres. La construction médiatique de multiples questions publiques emprunte la voie de l'individualisation des enjeux et des problèmes. C'est le cas en matière de comportement automobile (Gusfield, 1963), ou de santé (Romeyer, 2007). Quitte à ce que des questions d'insécurité routière, de lutte contre le cancer ou de garde d'enfants malades mettent prioritairement en avant les enjeux moraux et individuels (faire attention à sa santé, lutter contre l'alcool au volant), indépendamment de toutes considérations économiques, culturelles ou sociales (état des véhicules, place accordée à la prévention en matière de santé, etc.) favorisant ou facilitant ces 
comportements individuels (Devillard et Marchetti, 2008, 161).

\section{3. Conclusion}

La construction du don de RTT comme problème public a bénéficié d'actions de promotion et de relais de multiples acteurs : bénéficiaires, salariés donateurs, élus politiques, journalistes... Et pourtant, la médiatisation dont ces coopérations salariales ont fait l'objet entre 2009 et 2015 a donné de cette question une version réductrice. En effet, les cadrages médiatiques réduisent, au fur et à mesure des années, la place accordée aux discussions relatives aux enjeux politiques et philosophiques de ces actions au profit de la promotion de leurs dimensions individuelles et psychologiques. Cette médiatisation se traduit alors par un effacement rapide des enjeux (politiques, philosophiques, managériaux) inhérents à ces nouvelles formes de coopérations au profit des enjeux humains et sociaux. Pourtant, chronologiquement, la prise en charge politique de cette question par un député a été primordiale. De manière singulière, et contrairement à l'ordre des étapes décrites par Blumer ${ }^{19}$, la phase des réactions politiques a largement devancé celle de la publicisation élargie et de la légitimation de cette question. Ce constat oblige - dans ce cas - d'une part à relativiser (ou à préciser) l'importance d'une notion comme celle d'agenda setting tant, en l'espèce, l'agenda médiatique a suivi l'agenda politique. II oblige d'autre part à préciser l'importance de la notion de cadrage médiatique. Si les journalistes sont restés dépendants de leurs sources dans le choix de ce sujet, leur latitude - vis-à-vis du pouvoir politique tout au moins - reste tangible dans le traitement qu'ils en font, compte tenu de l'amplification (Nollet, 2010) opérée de points de vue personnels des salariés au détriment de considérations plus ouvertement politiques.

19 Sa description des étapes de promotion d'un fait social en problème est la suivante : la nomination du problème, sa publicisation, puis sa légitimation par des groupes aux intérêts divergents, et enfin la formation d'un consensus sur la définition du problème. Les réactions politiques viennent après et se traduisent par des plans d'actions (Blumer, 1971). 
Bibliographie

Blumer H. (1971). "Social problems as collective behavior ». In Social problems, vol. 18, p. 298-306.

Chasserio S. et Legault M.-J. (2005). «Dans la nouvelle économie, la conciliation entre la vie privée et la vie professionnelle passe par... l'augmentation des heures de travail ». In Recherches Sociographiques, vol. 46, n¹, p. 119-142.

Cobb R. and Ross M. (1997). « Agenda Setting and the Denial of Agenda Access: Key Concepts ". In Cobb R. and Ross M. (dirs.) Cultural Strategies of Agenda Denial: Avoidance, Attack and Redefinition, Laurence, University Press of Kansas, p. 3-23.

Comby J.-B. et Grossetête M. (2007). "De l'individualisation des problèmes publics aux lectures des enjeux ". In La médiatisation du politique, Congrès AFSP, Toulouse, $35 \mathrm{p}$.

Devillard V. et Marchetti D. (2008). "La 'sécurité routière', un programme sans risque. La neutralisation d'un problème politique et social à la télévision ». In Réseaux, n¹47, p. 149-176.

Freeman R. E. (1984). Strategic Management: A Stakeholder Approach, Boston, Pitman.

Galinski E., Bond J. T. and Friedman D. E. (1993). The Changing Workforce: Highlights of the
National Sudy, New York, Families and Work Institute.

Gusfield J. (1963). Symbolic Crusade. Status Politics and the American Temperance Movement, Champaign, University of Illinois Press.

Hall S. (2007). Identités et Cultures. Politiques des Cultural Studies, édité par M. Cervulle, trad. de C. Jacquet, Paris, Éditions Amsterdam.

Hassenteufel P. (2010). « Les processus de mise sur agenda : sélection et construction des problèmes publics ". In Informations sociales, $n^{\circ} 157$, p. 50-58.

Juhem P. (2001). " Luttes partisanes et fluctuation des cadres cognitifs des journalistes ». In Gerstlé J. (dir.), Les effets d'information en politique, Paris, L'Harmattan, p. 9-139.

Le Loarne-Lemaire S. et Sanseau P.Y., Smith M. (2014). « Reconsidérer le débat sur le rapport vie professionnelle/vie privée en France pour le dépasser : plaidoyer pour une approche relationnelle ». In RIMHE, vol. 2, $\mathrm{n}^{\circ} 12$, p. 41-51.

Macé É. (2005). « Les faits divers de 'violence urbaine' : effets d'agenda et de cadrage journalistique ". In Les cahiers du journalisme, $n^{\circ} 14$, p. 188-201.

Mainil D. (2015). La problématique du cadrage dans le traitement médiatique de l'État d'Israël. Analyse comparée des quotidiens Le Soir et Libération, Bruxelles, Étude annuelle. In Revue Regard, p. 1-59. 
Mercier A. (2012). "L'utile fiction de l'opinion publique ». In Mercier A. (dir.), Médias et opinion publique, Paris, CNRS, p. 15-40.

Mitchell R. K., Agle B. R. and Wood D. (1997). " Toward a Theory of Stakeholder Identification and Salience: Defining the Principle of Who and What Really Counts ". In Academy of Management Review, vol. $22, n^{\circ} 4$, p. 853-886.

Neuman W. R., Just M. and Crigler A. N. (1992), Common Knowledge: News and the Construction of Political Meaning, Chicago, Londres, University of Chicago Press.

Neveu É. (1999). « L'approche constructiviste des 'problèmes publics'. Un aperçu des travaux anglo-saxons ». In Études de communication, $n^{\circ} 22$, p. 41-58.

Neveu É. (2015). Sociologie politique des problèmes publics, Paris, Armand Colin.

Nollet J. (2010). Des décisions publiques "médiatiques " ? Sociologie de l'emprise du journalisme sur les politiques de sécurité sanitaire des aliments, Thèse de doctorat, Université Lille 2.

Quéré L. (1995). « La télévision régie-t-elle l'espace public ? ». In Esquenazi J.-P. (dir.), La communication de l'information, Paris, L'Harmattan, p. 10-28.

Romeyer H. (2007). « La santé à la télévision : émergence d'une question sociale ». In Questions de communication, $n^{\circ} 11$, p. 51-70.

Rouquette S. (2010). L'hypermédia internet, Paris, Ina Éditions.

Wolton D. (1997). Penser la communication, Paris, Flammarion. 
